\title{
Effect of Quasiparticles Injection on the AC Response of a Superconductor
}

\author{
G. Catelani and L. I. Glazman \\ Departments of Physics and Applied Physics, Yale University, New Haven, Connecticut 06520, USA \\ K. E. Nagaev, \\ Institute of Radioengineering and Electronics, Mokhovaya 11-7, Moscow, 125009 Russia
}

(Dated: November 20, 2018)

\begin{abstract}
We calculate the AC linear response of a superconductor in a nonequilibrium electronic state. The nonequilibrium state is produced by injecting quasiparticles into the superconductor from normal leads through asymmetric tunnel contacts. The dissipative part of the response is drastically increased by the injected quasiparticles and is proportional to their total number regardless of the imbalance between the numbers of electron-like and hole-like excitations.
\end{abstract}

PACS numbers: 74.25.N-,74.40.Gh

\section{INTRODUCTION}

Over the years, measurement of the impedance was used extensively in the studies of spectra of elementary charge carriers in normal metals and superconductors. In the case of normal-metal single crystals, measurements of surface impedance in conditions of cyclotron resonance were instrumental in the reconstruction of the Fermi surface geometry $\stackrel{\underline{1}}{\underline{1}}$ In superconductors, the temperature dependence of the impedance allowed one to investigate the appearance of the BCS gap in the spectrum

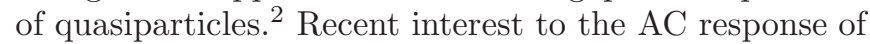
superconductors is driven by its application for studying new materials ${ }^{3.4}$ and by the use of well-studied superconductors as material for high-quality resonators. Depending on the problem, it is important to realize the lowestpossible dissipation rate of a resonator, or its well-defined response to a perturbation. The former goal is central for superconducting qubit physics $\frac{5,6}{5}$ and quantum optics,$\frac{7.8}{,}$ where one is interested in achieving the longest-possible coherence times. The latter one is important, e.g., for the use of superconductors in microwave kinetic inductance detectors $\underline{\underline{9}}$

To interpret the measured impedance, one has to compare it with theoretical predictions. However up to now, no microscopic calculations of impedance of superconductors under nonequilibrium conditions were available. The frequency and temperature dependence of the complex conductivity of a superconductor within the BCS theory was first evaluated in the seminal paper of Mattis and Bardeen, $\stackrel{10}{=}$ where the limit of short electron mean free path (the "dirty superconductor" limit) was considered. The non-local conductivity of a clean superconductor was derived by Abrikosov, Gorkov and Khalatnikov 11 Later works of Nam $\frac{12}{2}$ were aimed at bridging the two limiting cases. The theory developed in Refs. $10-12$ addresses superconductors at thermal equilibrium. If desired, this condition is fairly easy to achieve at not-too-low temperatures. Indeed, even the early measurements have demonstrated the hallmarks of the BCS behavior of the dissipative part of the impedance, including its thermalactivation behavior at sub-gap frequencies $\frac{13}{\underline{3}}$ At the same

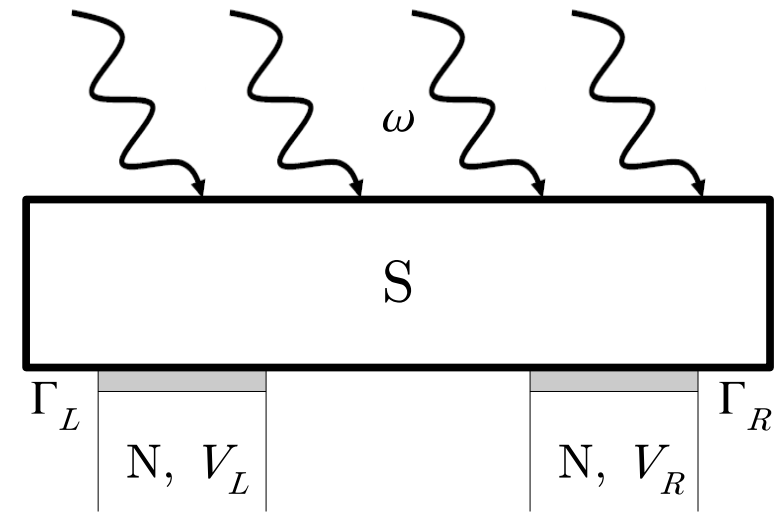

FIG. 1: N-I-S-I-N structure: the superconductor (S) is connected to two normal leads $(\mathrm{N})$ - maintained at different voltages (bias $V=V_{R}-V_{L}$ ) - via tunnel junctions characterized by tunneling rates $\Gamma_{R}, \Gamma_{L}$. The superconductor is also subject to a weak electromagnetic field oscillating with frequency $\omega$.

time, experiments involving impedance measurements in non-equilibrium conditions relied on heuristic extension of Mattis-Bardeen formula, amounting to the replacement of the equilibrium quasiparticle distribution function in the formula by a non-equilibrium one $, 9,14$

The goal of this paper is to microscopically evaluate the linear AC conductivity of a superconductor in a concrete setup allowing a controlled perturbation of the quasiparticle distribution function. To this end, we analyze the steady state and linear AC conductivity of a superconductor brought out of equilibrium by electron tunneling through two junctions connecting the superconductor to normal leads (N-I-S-I-N structure - see Fig. 11); the structure is biased by a constant voltage $V$.

We find the steady-state electron distribution at finite temperature $T$ and voltage $V$, assuming these two scales small compared to the quasiparticle energy gap $\Delta$. In the case of unequal conductances of the two tunnel junctions, charge imbalance is created along with a perturbation of the energy distribution of quasiparticles. We evaluate 
the complex AC conductivity of the superconductor in this non-equilibrium state and show that only the energy mode of the quasiparticle distribution enters in the proper generalization of the Mattis-Bardeen formula. At $e V, k_{B} T \ll \Delta$ we cast the result for conductivity in terms of $T$ and quasiparticle density $n_{q p}$. That form extrapolates between the equilibrium result (where $n_{q p}$ is a function of $T$ only) and the non-equilibrium one, where $n_{q p}$ is a function of $V$ and $T$, with arbitrary ratio $e V / k_{B} T$.

In the next Section, we formulate the problem in terms of matrix Green functions. The steady state of the electrons in the superconductor formed in the presence of finite bias applied to the N-I-S-I-N structure is found in Section III where we also establish the correspondence between the descriptions in terms of the matrix distribution function $\hat{F}(\varepsilon)$ and the scalar distribution function of quasiparticles $f_{\xi}$ (the latter was used in the original Mattis-Bardeen theory for the AC conductivity at equilibrium). Using the description in terms of $f_{\xi}$, we include the electron-phonon interaction into the consideration of quasiparticle kinetics. The $\mathrm{AC}$ conductivity at low temperature and bias (but at arbitrary $e V / k_{B} T$ ) is analyzed in Section [V] Throughout the paper, we use units $\hbar=k_{B}=1$.

\section{ELECTRON DYNAMICS IN A SUPERCONDUCTOR SUBJECT TO DC BIAS AND WEAK AC FIELD}

We consider a diffusive superconductor connected to two normal leads, left $(L)$ and right $(R)$, via tunnel barriers and exposed to an external, time-dependent electric field. The system properties can be described in terms of disorder-averaged matrix Green's functions for the superconductor, $\check{\mathcal{G}}_{S}\left(\mathbf{k}, t, t^{\prime}\right)$, and for the electrodes, $\check{\mathcal{G}}_{i}\left(\mathbf{p}_{i}, t, t^{\prime}\right)$, $i=L, R$. Each of the matrices $\breve{\mathcal{G}}$ has the form, in Keldysh space $\frac{15}{}$

$$
\check{\mathcal{G}}=\left(\begin{array}{cc}
\hat{\mathcal{G}}^{R} & \hat{\mathcal{G}}^{K} \\
0 & \hat{\mathcal{G}}^{A}
\end{array}\right)
$$

The elements of this matrix are $2 \times 2$ matrices in Nambu particle-hole space. The superconductor Green's function obeys the Dyson equation

$$
\begin{aligned}
& {\left[i \hat{\tau}_{z} \frac{\partial}{\partial t}-\left(\frac{(\mathbf{k}-e \mathbf{A}(t))^{2}}{2 m}-E_{F}\right)+i \hat{\tau}_{y} \Delta\right] \check{\mathcal{G}}_{S}\left(\mathbf{k}, t, t^{\prime}\right)} \\
& \quad-\int d t^{\prime \prime} \frac{1}{2 \pi \tau \nu_{S}} \sum_{\mathbf{k}^{\prime}} \check{\mathcal{G}}_{S}\left(\mathbf{k}^{\prime}, t, t^{\prime \prime}\right) \check{\mathcal{G}}_{S}\left(\mathbf{k}, t^{\prime \prime}, t^{\prime}\right) \\
& =\hat{1} \delta\left(t-t^{\prime}\right)+\int d t^{\prime \prime} \sum_{i, \mathbf{p}_{i}} T_{i}^{2} \check{\mathcal{G}}_{i}\left(\mathbf{p}_{i}, t, t^{\prime \prime}\right) \check{\mathcal{G}}_{S}\left(\mathbf{k}, t^{\prime \prime}, t^{\prime}\right)
\end{aligned}
$$

Here $\mathbf{A}(\mathbf{t})$ is the vector potential, which is related to the electric field via $\mathbf{E}=-\partial \mathbf{A} / \partial t$. The coefficient $1 / \tau$ is the impurity scattering rate, and $\nu_{S}$ is the density of states at the Fermi level in the superconductor. The matrix element $T_{i}$ for tunneling into lead $i$ determines the dimensionless conductance $\mathrm{g}_{i}=8 \pi^{2} \nu_{S} \nu_{i} T_{i}^{2}$ of junction $i$, where $\nu_{i}$ is the density of states in the lead. The assumption

$$
\mathrm{g}_{L}+\mathrm{g}_{R} \ll \mathrm{g}_{S}
$$

where $\mathrm{g}_{S}$ is the normal-state conductance of the superconductor, justifies the use of the tunneling Hamiltonian from which the last term in Eq. (2) is derived. The same assumption enables us to neglect small spatial variations of the order parameter, which we take to be uniform, real, and time-independent. The last two conditions amount to a choice of gauge.

Since we are interested in the linear response to the external field, we first consider the system in the absence of field, but with the leads at different potentials. This is the focus of the next section.

\section{NON-EQUILIBRIUM STEADY STATE}

When there is no external field $(\mathbf{A}=0)$, the Green's functions are isotropic in momentum space and depend on the the difference $t-t^{\prime}$. Then the semiclassical Green's functions

$$
\check{G}\left(t-t^{\prime}, \mathbf{n}\right)=\frac{i}{\pi} \int d \xi_{\mathbf{p}} \check{\mathcal{G}}\left(\mathbf{p}, t-t^{\prime}\right), \quad \mathbf{n}=\frac{\mathbf{p}}{|\mathbf{p}|},
$$

where $\xi_{\mathbf{p}}=p^{2} / 2 m-E_{F}$, depend only on the time difference, and not on the momentum direction $\mathbf{n}$. Therefore, we omit the argument $\mathbf{n}$ for the rest of this section. In the normal leads, the elements of $\check{G}_{i}$ are (in the frequency domain)

$$
\begin{aligned}
\hat{G}_{i}^{R} & =\hat{\tau}_{z}, \quad \hat{G}_{i}^{A}=-\hat{\tau}_{z}, \\
\hat{G}_{i}^{K} & =\hat{G}_{i}^{R} \hat{n}_{i}-\hat{n}_{i} \hat{G}_{i}^{A},
\end{aligned}
$$

with

$$
\hat{n}_{i}(\varepsilon)=\left(\begin{array}{cc}
n\left(\varepsilon-e V_{i}\right) & 0 \\
0 & n\left(\varepsilon+e V_{i}\right)
\end{array}\right), n(\varepsilon)=\tanh \left(\frac{\varepsilon}{2 T}\right) .
$$

The potentials $V_{i}$ in the leads are measured from the superconductor chemical potential. Using Eq. (5), Eq. (6) can be rewritten as

$$
\hat{G}_{i}^{K}=2 n_{i}^{0} \hat{\tau}_{z}+2 n_{i}^{1} \hat{1}
$$

where

$$
\begin{aligned}
& n_{i}^{0}=\frac{1}{2}\left[n\left(\varepsilon-e V_{i}\right)+n\left(\varepsilon+e V_{i}\right)\right], \\
& n_{i}^{1}=\frac{1}{2}\left[n\left(\varepsilon-e V_{i}\right)-n\left(\varepsilon+e V_{i}\right)\right] .
\end{aligned}
$$

The superconductor Green's function $\check{G}_{s}$ is determined by Eq. (2); taking the difference between Eq. (2) and its conjugate, and using Eqs. (4), we find

$$
\left[\varepsilon \hat{\tau}_{z}+i \Delta \hat{\tau}_{y}, \check{G}_{S}\right]=-i\left[\Gamma_{L} \check{G}_{L}+\Gamma_{R} \check{G}_{R}, \check{G}_{S}\right]
$$


where $\Gamma_{i}=\pi \nu_{i} T_{i}^{2}$ are the tunneling rates and $\nu_{i}$ are the densities of states in the leads $i=L, R$. This equation must be supplemented by the normalization condition, 16 which in the frequency domain takes the form

$$
\check{G}_{S}(\varepsilon) \check{G}_{S}(\varepsilon)=\hat{1} .
$$

Isolating the $R(A)$ component of Eq. (10) gives

$$
\hat{H}^{R(A)} \hat{G}_{S}^{R(A)}-\hat{G}_{S}^{R(A)} \hat{H}^{R(A)}=0,
$$

where

$$
\hat{H}^{R(A)}=\left(\varepsilon \pm i \Gamma_{L} \pm i \Gamma_{R}\right) \hat{\tau}_{z}+i \Delta \hat{\tau}_{y} .
$$

In view of the $R(A)$ component of the normalization condition (11), $\left(\hat{G}_{S}^{R(A)}\right)^{2}=\hat{1}$, the solution to Eq. (12) can be written as

$$
\hat{G}_{S}^{R(A)}=\hat{H}^{R(A)} / \tilde{\xi}^{R(A)},
$$

where

$$
\tilde{\xi}^{R(A)}= \pm\left[\left(\varepsilon \pm i \Gamma_{L} \pm i \Gamma_{R}\right)^{2}-\Delta^{2}\right]^{1 / 2} .
$$

In the limit $\Gamma_{i} \rightarrow 0, \mathrm{Eq}$. (14) reduces to the well-known semiclassical BCS expression 16 When $\Gamma_{i} \neq 0$ the excitations in the superconductor have finite lifetime due to tunneling into the normal leads and this causes broadening of the density of states $\frac{17}{1 n}$ what follows we assume that the broadening is much smaller than the gap, $\Gamma_{L}+\Gamma_{R} \ll \Delta$. Since the dimensionless conductance, as defined in the text before Eq. (3), equals $g_{i}=8 \pi \Gamma_{i} / \delta$, with $\delta$ being the level spacing in the superconductor, we can express this condition as

$$
\mathrm{g}_{L}+\mathrm{g}_{R} \ll \Delta / \delta .
$$

We now consider the Keldysh component of Eq. (10). It may be written in the form

$$
\begin{aligned}
\hat{H}^{R} \hat{G}_{S}^{K}-\hat{G}_{S}^{K} \hat{H}^{A}= & -i \Gamma_{L}\left(\hat{G}_{L}^{K} \hat{G}_{S}^{A}-\hat{G}_{S}^{R} \hat{G}_{L}^{K}\right) \\
& -i \Gamma_{R}\left(\hat{G}_{R}^{K} \hat{G}_{S}^{A}-\hat{G}_{S}^{R} \hat{G}_{R}^{K}\right)
\end{aligned}
$$

Its left-hand side can be simplified as follows: first, we use Eq. (14) to rewrite $\hat{H}^{R(A)}$ in terms of $\hat{G}_{S}^{R(A)}$; then we replace the advanced Green's function with the retarded one employing the orthogonality condition [the Keldysh component of Eq. [11)]

$$
\hat{G}_{S}^{R} \hat{G}_{S}^{K}+\hat{G}_{S}^{K} \hat{G}_{S}^{A}=0 .
$$

Multiplying the resulting equation by $\hat{G}_{S}^{R}$ from the left and using again $\left(\hat{G}_{S}^{R}\right)^{2}=\hat{1}$, we arrive at

$$
\begin{aligned}
\left(\tilde{\xi}^{R}+\tilde{\xi}^{A}\right) \hat{G}_{S}^{K} & =i \Gamma_{L}\left(\hat{G}_{L}^{K}-\hat{G}_{S}^{R} \hat{G}_{L}^{K} \hat{G}_{S}^{A}\right) \\
& +i \Gamma_{R}\left(\hat{G}_{R}^{K}-\hat{G}_{S}^{R} \hat{G}_{R}^{K} \hat{G}_{S}^{A}\right)
\end{aligned}
$$

If $|\varepsilon|<\Delta$, the sum $\tilde{\xi}^{R}+\tilde{\xi}^{A}$ is of the order of $\Delta$, i. e. much larger than $\Gamma_{L}$ and $\Gamma_{R}$. However, if $|\varepsilon|>\Delta$ this quantity is proportional to these small parameters and can be approximated as

$$
\tilde{\xi}^{R}+\tilde{\xi}^{A} \approx i\left(\Gamma_{R}+\Gamma_{L}\right) \varepsilon\left(\frac{1}{\xi^{R}}-\frac{1}{\xi^{A}}\right),
$$

where

$$
\xi^{R(A)}= \pm\left[\left(\varepsilon \pm i 0^{+}\right)-\Delta^{2}\right]^{1 / 2}
$$

Consistently with this approximation, the Green's functions $G_{S}^{R / A}$ in the right-hand side of Eq. (17) should be taken at zero order in $\Gamma_{i}$ 's. Using Eqs. (8) and (14), after some algebra we obtain

$$
\hat{G}_{S}^{K}=\left(\frac{1}{\xi^{R}}-\frac{1}{\xi^{A}}\right)\left[n^{0}\left(\varepsilon \hat{\tau}_{z}+i \Delta \hat{\tau}_{y}\right)+n^{1} \frac{\varepsilon^{2}-\Delta^{2}}{\varepsilon}\right],
$$

where $(a=0,1)$

$$
n^{a}=\frac{\Gamma_{L} n_{L}^{a}+\Gamma_{R} n_{R}^{a}}{\Gamma_{L}+\Gamma_{R}}
$$

with $n_{L, R}^{a}$ of Eq. (9). We stress that Eqs. (19)-(21) are not restricted to junctions with conductances smaller than the conductance quantum, the conditions of applicability being given by Eqs. (3) and (16).

The matrix $\hat{G}_{S}^{K}$ depends on the voltages $V_{L}, V_{R}$ via Eqs. (9) and (22). However, under steady-state conditions these two quantities are not independent and, as we discuss in the next section, the state of the superconductor is fully determined by their difference $V=V_{R}-V_{L}$.

\section{A. Current and potentials}

The results of the previous sections rest on the steadystate assumption. It means that the total current flowing out of the superconductor must vanish. This requirement, as we show below, defines the division of the applied bias between the two tunnel junctions connecting the superconductor to the normal leads.

The current leaving through the left tunnel contact is given by

$$
\begin{aligned}
I_{L}=\frac{1}{4} e \nu_{S} \Gamma_{L} \int d \varepsilon \operatorname{Tr} & {\left[\hat { \tau } _ { z } \left(\hat{G}_{L}^{R} \hat{G}_{S}^{K}+\hat{G}_{L}^{K} \hat{G}_{S}^{A}\right.\right.} \\
& \left.\left.-\hat{G}_{S}^{R} \hat{G}_{L}^{K}-\hat{G}_{S}^{K} \hat{G}_{L}^{A}\right)\right] .
\end{aligned}
$$

The current $I_{R}$ into the right contact is found by replacing $L \rightarrow R$. Therefore the total outgoing current is

$$
I_{L}+I_{R}=e \nu_{S} \int d \varepsilon\left(\frac{1}{\xi^{R}}-\frac{1}{\xi^{A}}\right) \frac{\Delta^{2}}{\varepsilon}\left(\Gamma_{L} n_{1 L}+\Gamma_{R} n_{1 R}\right)
$$


and must be zero under steady-state conditions, as discussed above. [We used Eqs. (8), (14), and (21) in derivation of Eq. (24).] In other words, the requirement

$$
I_{L}+I_{R}=0
$$

determines the relationship between the voltages $V_{L}, V_{R}$, that enter as parameters in Eq. (22).

Let us consider explicitly the regime of low temperatures and voltages so that $\max \left\{e V_{L}, e V_{R}, T\right\} \ll \Delta$. Then the following approximate expression for the functions $n_{L(R)}^{1}$ are valid at energies $|\varepsilon|>\Delta$ :

$$
n_{i}^{1}(\varepsilon) \simeq-2 \sinh \left(e V_{i} / T\right) \exp (-|\varepsilon| / T) .
$$

Substituting into Eqs. (24)-(25) we obtain the condition

$$
\Gamma_{L} \sinh \left(e V_{L} / T\right)+\Gamma_{R} \sinh \left(e V_{R} / T\right)=0 .
$$

Introducing the bias $V=V_{R}-V_{L}$, we find from this equation that

$$
\sinh \left(e V_{L} / T\right)=-\frac{\Gamma_{R} \sinh (e V / T)}{\sqrt{\Gamma_{L}^{2}+\Gamma_{R}^{2}+2 \Gamma_{L} \Gamma_{R} \cosh (e V / T)}} .
$$

Thanks to this relation, we can reexpress voltages $V_{L}, V_{R}$ in terms of the bias $V$, and the non-equilibrium steady state in the limit $e V, T \ll \Delta$ is fully determined by these quantities.

\section{B. Quasiparticle distribution function}

In the preceding sections we have found an explicit expression for the non-equilibrium Keldysh Green's function for the biased superconductor, Eq. (21). In this section we derive the relationship between the Keldysh Green's function and the distribution function $f$ for the Bogoliubov quasiparticles.

Our starting point is the definition of $\hat{G}_{S}^{K}$ (in the time domain) in terms of creation and annihilation operators

$$
\begin{aligned}
& \hat{G}_{S}^{K}\left(t, t^{\prime}\right) \\
& =\frac{\hat{\tau}_{z}}{\pi \nu_{S}} \sum_{\mathbf{p}}\left(\begin{array}{ll}
\left\langle\left[c_{m \uparrow}(t), c_{m \uparrow}^{\dagger}\left(t^{\prime}\right)\right]\right\rangle & \left\langle\left[c_{m \uparrow}(t), c_{m \downarrow}\left(t^{\prime}\right)\right]\right\rangle \\
\left\langle\left[c_{m \downarrow}^{\dagger}(t), c_{m \uparrow}^{\dagger}\left(t^{\prime}\right)\right]\right\rangle & \left\langle\left[c^{\dagger}(t)_{m \downarrow}, c_{m \downarrow}\left(t^{\prime}\right)\right]\right\rangle
\end{array}\right) .
\end{aligned}
$$

The sum over single-particle states $\frac{1}{\nu_{S}} \sum_{m}$ with energy $\xi_{m}$ is equivalent to the integration over their energy $\xi_{\mathbf{p}}$ in Eq. (4). Next, we perform the Bogoliubov transformation from electron operators $c_{m \uparrow}, c_{m \downarrow}$ to quasiparticle operators $\alpha_{m}, \beta_{m}$ :

$$
c_{m \uparrow}=u_{m} \alpha_{m}+v_{m} \beta_{m}^{\dagger}, \quad c_{m \downarrow}=u_{m} \beta_{m}-v_{m} \alpha_{m}^{\dagger}
$$

with amplitudes $u_{m}, v_{m}$ given by

$$
\begin{aligned}
& \left|u_{m}\right|^{2}=1-\left|v_{m}\right|^{2}=\frac{1}{2}\left(1+\frac{\xi_{m}}{\epsilon_{m}}\right), \\
& u_{m} v_{m}=-\frac{1}{2} \frac{\Delta}{\epsilon_{m}}
\end{aligned}
$$

and $\epsilon_{m}=\sqrt{\xi_{m}^{2}+\Delta^{2}}$ being the quasiparticle energy. Introducing the distribution function (we assume equal population of the two spins)

$$
f\left(\xi_{m}\right)=\left\langle\alpha_{m}^{\dagger} \alpha_{m}\right\rangle=\left\langle\beta_{m}^{\dagger} \beta_{m}\right\rangle,
$$

using Eq. (31), and taking the Fourier transform with respect to $t-t^{\prime}$ we obtain

$$
\begin{aligned}
\hat{G}_{S}^{K}(\varepsilon)=2 \operatorname{Re} \frac{1}{\sqrt{\varepsilon^{2}-\Delta^{2}}}\left\{\left(\varepsilon \hat{\tau}_{z}+i \Delta \hat{\tau}_{y}\right)\left[1-2 f^{E}(\varepsilon)\right]\right. \\
\left.-2 \sqrt{\varepsilon^{2}-\Delta^{2}} f^{Q}(\varepsilon)\right\}
\end{aligned}
$$

with the energy and charge modes of the distribution function $f\left(\xi_{m}\right)$ defined as

$$
\begin{gathered}
f^{E}(\varepsilon)=\frac{1}{2}\left[f\left(\sqrt{\varepsilon^{2}-\Delta^{2}}\right)+f\left(-\sqrt{\varepsilon^{2}-\Delta^{2}}\right)\right], \\
f^{Q}(\varepsilon)=\frac{1}{2}\left[f\left(\sqrt{\varepsilon^{2}-\Delta^{2}}\right)-f\left(-\sqrt{\varepsilon^{2}-\Delta^{2}}\right)\right] .
\end{gathered}
$$

Hence the Keldysh Green's function can be presented in the form

$$
\hat{G}_{S}^{K}=\hat{G}_{S}^{R} \hat{F}-\hat{F} \hat{G}_{S}^{A}
$$

where

$$
\begin{aligned}
\hat{F}= & \operatorname{sgn} \varepsilon\left[1-2 f^{E}(\varepsilon)\right] \hat{1} \\
& -\frac{|\varepsilon|}{\sqrt{\varepsilon^{2}-\Delta^{2}}}\left(\hat{\tau}_{z}+i \frac{\Delta}{\varepsilon} \hat{\tau}_{y}\right) 2 f^{Q}(\varepsilon) .
\end{aligned}
$$

This shows that the trace of $\hat{F}$ is directly related to the energy mode $f^{E}$, while the traceless part possesses an additional density of states factor compared to the charge mode $f^{Q}$ of the quasiparticle distribution function.

Comparison of Eq. (36) with the solution of the kinetic equation for $\hat{G}_{S}^{K}$, Eq. (21), enables us to find the explicit expressions for the distribution function modes

$$
\begin{aligned}
& f^{E}(\varepsilon)=\frac{1}{2}\left[1-\operatorname{sgn} \varepsilon n^{0}(\varepsilon)\right] \\
& f^{Q}(\varepsilon)=-\frac{1}{2|\varepsilon|} \sqrt{\varepsilon^{2}-\Delta^{2}} n^{1}(\varepsilon)
\end{aligned}
$$

and the voltages $V_{L}, V_{R}$ are related by Eq. (25). At low temperature and bias $T, e V \ll \Delta$, at leading order in $e^{-\Delta / T}$ we obtain, using Eq. (28),

$$
f^{E}(\varepsilon) \approx \Phi(e V / T) e^{-\varepsilon / T},
$$

with

$$
\Phi(e V / T)=\frac{1}{\Gamma_{L}+\Gamma_{R}} \sqrt{\Gamma_{L}^{2}+\Gamma_{R}^{2}+2 \Gamma_{L} \Gamma_{R} \cosh (e V / T)},
$$

while at this order $f^{Q}$ vanishes due to Eq. (27).

One may also obtain the distribution function $f_{\xi}$ by solving the kinetic equation for quasiparticles. The latter method is convenient to assess the effects of relaxation. In the next section we consider in more detail the electron-phonon interaction. 


\section{Kinetic equation for quasiparticles}

In the kinetic equation approach, the effects of interaction are described by adding to the rate of change of the occupation numbers due to tunneling an appropriate collision integral $I$. In the case of electron-phonon interaction, one can derive a set of coupled kinetic equations for the two modes of the quasiparticle distribution function, $f^{E}$ and $f^{Q}$ :

$$
\begin{aligned}
\frac{d}{d t} f^{E} & =\Gamma_{L} f_{0 L}+\Gamma_{R} f_{0 R}-\Gamma f^{E} \\
& +I_{r}^{E}\left\{f^{E}, f^{Q}, N\right\}+I_{s}^{E}\left\{f^{E}, f^{Q}, N\right\}, \\
\frac{d}{d t} f^{Q} & =-\frac{\sqrt{\varepsilon^{2}-\Delta^{2}}}{\varepsilon}\left[\Gamma_{L} f_{1 L}+\Gamma_{R} f_{1 R}\right]-\Gamma f^{Q} \\
& +I_{r}^{Q}\left\{f^{E}, f^{Q}, N\right\}+I_{s}^{Q}\left\{f^{E}, f^{Q}, N\right\},
\end{aligned}
$$

where $\Gamma=\Gamma_{L}+\Gamma_{R}, f_{0 i}=\left(1-n_{i}^{0}\right) / 2$, and $f_{1 i}=n_{i}^{1} / 2$. Neglecting the collision integrals, in the steady-state $d f / d t=0$ we recover immediately the result Eq. (37). The subscripts $r$ and $s$ are used to distinguish, in the electron-phonon collision integrals, quasiparticle recombination and scattering processes, respectively, and we take the phonon distribution function $N$ to be the thermal equilibrium one:

$$
N_{\varepsilon}=\frac{1}{e^{\varepsilon / T}-1} .
$$

We characterize the electron-phonon collision rate by the scattering rate, $1 / \tau_{p h}$, for normal electrons off phonons at the critical temperature $T_{c}, 18$ We consider the limit of low temperature and bias, in which case the electron-phonon interaction has a small effect on the quasiparticle distribution as long as tunneling is the main scattering process. Considering relaxation rate for an arbitrary small perturbation 19 of the quasiparticle distribution function, one might conclude that the corresponding condition for that is $\left(1 / \tau_{p h}\right)(T / \Delta)^{7 / 2} \ll \Gamma$. (The small factor $(T / \Delta)^{7 / 2}$ here suppresses the low temperature scattering rate for quasiparticles compared to that of normal electrons at $T_{c}$.) However, for the specific case of out-of-equilibrium distribution created by tunneling the condition for the rate $1 / \tau_{p h}$ is even softer, see Eq. (50) below.

Evaluating the correction to the charge mode $f^{Q}$ due to the weak electron-phonon relaxation at low temperatures, we find that such correction to Eq. (37) vanishes at leading order in $e^{-\Delta / T}$ due to the relation Eq. (27).

We now consider the energy mode. The collision integrals $I_{r, s}^{E}$ contain terms quadratic in $f^{Q}$, which we can neglect since $f^{Q}$ vanishes at leading order, as discussed above. The non-vanishing terms are ${ }^{20}$

$$
\begin{array}{r}
I_{r}^{E}=\frac{1}{\tau_{p h} \Delta^{3}} \int_{\Delta}^{\infty} d \varepsilon^{\prime} \frac{\varepsilon^{\prime}}{\sqrt{\varepsilon^{\prime 2}-\Delta^{2}}}\left(\varepsilon+\varepsilon^{\prime}\right)^{2}\left(1+\frac{\Delta^{2}}{\varepsilon \varepsilon^{\prime}}\right) \\
{\left[\left(1-f_{\varepsilon}^{E}\right)\left(1-f_{\varepsilon^{\prime}}^{E}\right) N_{\varepsilon+\varepsilon^{\prime}}-f_{\varepsilon}^{E} f_{\varepsilon^{\prime}}^{E}\left(N_{\varepsilon+\varepsilon^{\prime}}+1\right)\right]}
\end{array}
$$

for recombination processes and

$$
\begin{aligned}
& I_{s}^{E}=\frac{1}{\tau_{p h} \Delta^{3}}\left\{\int_{\Delta}^{\varepsilon} d \varepsilon^{\prime} \frac{\varepsilon^{\prime}}{\sqrt{\varepsilon^{\prime 2}-\Delta^{2}}}\left(\varepsilon-\varepsilon^{\prime}\right)^{2}\left(1-\frac{\Delta^{2}}{\varepsilon \varepsilon^{\prime}}\right)\right. \\
& {\left[\left(1-f_{\varepsilon}^{E}\right) f_{\varepsilon^{\prime}}^{E} N_{\varepsilon-\varepsilon^{\prime}}-f_{\varepsilon}^{E}\left(1-f_{\varepsilon^{\prime}}^{E}\right)\left(N_{\varepsilon-\varepsilon^{\prime}}+1\right)\right]} \\
& +\int_{\varepsilon}^{\infty} d \varepsilon^{\prime} \frac{\varepsilon^{\prime}}{\sqrt{\varepsilon^{\prime 2}-\Delta^{2}}}\left(\varepsilon-\varepsilon^{\prime}\right)^{2}\left(1-\frac{\Delta^{2}}{\varepsilon \varepsilon^{\prime}}\right) \\
& \left.\left[\left(1-f_{\varepsilon}^{E}\right) f_{\varepsilon^{\prime}}^{E}\left(N_{\varepsilon^{\prime}-\varepsilon}+1\right)-f_{\varepsilon}^{E}\left(1-f_{\varepsilon^{\prime}}^{E}\right) N_{\varepsilon^{\prime}-\varepsilon}\right]\right\}
\end{aligned}
$$

for scattering processes.

We solve the kinetic equation (40) by iterations, writing $f^{E}$ in the form:

$$
f^{E} \simeq f^{E(0)}+\frac{1}{\tau_{p h} \Gamma} f^{E(1)}
$$

with $f^{E(0)}$ given in Eq. (38). At leading order in $e^{-\Delta / T}$, only the scattering collision integral $I_{s}^{E}$ is present. It is satisfied by any Boltzmann distribution function, like the one in Eq. (38). Indeed, let us consider the terms in square brackets in the second line of Eq. (44); in the low temperature and bias regime they are approximately

$$
\Phi e^{-\varepsilon^{\prime} / T} N_{\varepsilon-\varepsilon^{\prime}}-\Phi e^{-\varepsilon / T}\left(N_{\varepsilon-\varepsilon^{\prime}}+1\right)=0,
$$

in agreement with the detailed balance in the absence of recombination. Similarly, the terms in square brackets in the last line of Eq. (44) add up to zero. Therefore, at order $e^{-\Delta / T}$ there are no corrections to the distribution function.

To calculate the correction due to recombination, we note that $\varepsilon+\varepsilon^{\prime}>2 \Delta$, so that in the last term in square brackets in Eq. (43) we can neglect $N_{\varepsilon+\varepsilon^{\prime}}$ in comparison to unity. The square brackets then becomes approximately

$$
e^{-\left(\varepsilon+\varepsilon^{\prime}\right) / T}\left(1-\Phi^{2}\right)
$$

i.e., they are of order $e^{-2 \Delta / T}$. For the correction we find

$$
\begin{aligned}
f^{E(1)}= & e^{-\varepsilon / T}\left(1-\Phi^{2}\right) \frac{1}{\Delta^{3}} \int_{\Delta}^{\infty} d \varepsilon^{\prime} \frac{\varepsilon^{\prime}}{\sqrt{\varepsilon^{\prime 2}-\Delta^{2}}} \\
& \times\left(\varepsilon+\varepsilon^{\prime}\right)^{2}\left(1+\frac{\Delta^{2}}{\varepsilon \varepsilon^{\prime}}\right) e^{-\varepsilon^{\prime} / T} .
\end{aligned}
$$

After the substitution $\varepsilon^{\prime}=\Delta+T x$, the integral can be evaluated to give, at leading order

$$
f^{E(1)} \simeq e^{-\varepsilon / T}\left(1-\Phi^{2}\right) e^{-\Delta / T} \sqrt{\frac{\pi T}{2 \Delta}}\left(\frac{\varepsilon}{\Delta}\right)^{2}\left(1+\frac{\Delta}{\varepsilon}\right)^{3} .
$$

[Note that at zero bias $\Phi(0)=1$ and the correction vanishes.] From this equation we can estimate the ratio between the leading term and the correction in Eq. (45) and find that the iterative solution is applicable if

$$
\frac{1}{\tau_{p h} \Gamma} \ll \sqrt{\frac{\Delta}{T}} e^{\Delta / T}
$$


Under this condition, the electron-phonon relaxation leads only to a small modification of the distribution function given by Eqs. (45) and (49). Albeit the correction Eq. (49) at low energies $(\varepsilon \approx \Delta)$ scales with temperature predominantly as $e^{-2 \Delta / T}$, it may exceed the corresponding term of expansion of Eq. (37) in $e^{-\Delta / T}$. That happens at large values of $1 / \tau_{p h} \Gamma$, which still may satisfy Eq. (50).

This concludes our analysis of the non-equilibrium steady state of a superconductor for the specific problem of a finite-bias tunnel injection of quasiparticles. In the next section we consider the linear response of an outof-equilibrium superconductor to an external AC field.

\section{AC CONDUCTIVITY OF N-I-S-I-N STRUCTURE}

In this section we study the linear response of the superconductor to an external AC electric field oscillating at frequency $\omega$. Within linear response, the Green's function $G_{S}$ is the sum of the zero-approximation isotropic part $\check{G}_{0}$ and a small correction $\check{G}_{1}$, linear in A, which is anisotropic in momentum space

$$
\check{G}_{S}(\mathbf{n})=\check{G}_{0}+\check{G}_{1}(\mathbf{n}), \quad \mathbf{n}=\mathbf{p} /|\mathbf{p}| .
$$

The correction $\breve{G}_{1}(\mathbf{n})$ determines the current density via

$$
\mathbf{j}_{\omega}=-\frac{1}{4} e \nu_{S} \int_{-\infty}^{\infty} d \varepsilon \operatorname{Tr}\left\langle\mathbf{v} \hat{\tau}_{z} \hat{G}_{1}^{K}\right\rangle,
$$

where $\mathbf{v}=v_{F} \mathbf{n}, v_{F}$ is the Fermi velocity, and angular brackets denote averaging over the momentum direction. We emphasize that the calculation of the $\mathrm{AC}$ response that we present here is not restricted to the particular non-equilibrium state considered in the previous section; rather, it is valid for a generic matrix distribution function $\hat{F}$, which determines the Keldysh part of the zeroapproximation Green's function $\check{G}_{0}$ via Eq. (35).

The two terms in the sum (51) obey the orthogonality condition

$$
\check{G}_{+} \check{G}_{1}(\varepsilon, \omega)+\check{G}_{1}(\varepsilon, \omega) \check{G}_{-}=0,
$$

where

$$
\check{G}_{ \pm}=\check{G}_{0}(\varepsilon \pm \omega / 2) .
$$

An equation for $\check{G}_{1}$ can obtained as before by considering the difference between Eq. (2) and its conjugate and using Eqs. (41). Assuming $\mathbf{A}(t)=\mathbf{A}_{\omega} e^{-i \omega t}$ we find, at zeroth order in the tunneling rates $\Gamma_{i}$

$$
\begin{aligned}
-i \hat{H}_{+} \check{G}_{1}+i \check{G}_{1} \hat{H}_{-} & +\frac{1}{2 \tau} \check{G}_{+} \check{G}_{1}-\frac{1}{2 \tau} \check{G}_{1} \check{G}_{-} \\
& =i \operatorname{ve} \mathbf{A}_{\omega}\left(\hat{\tau}_{z} \check{G}_{-}-\check{G}_{+} \hat{\tau}_{z}\right),
\end{aligned}
$$

where

$$
\hat{H}_{ \pm}=(\varepsilon \pm \omega / 2) \hat{\tau}_{z}+i \Delta \hat{\tau}_{y}
$$

The zero-order in $\Gamma_{i}$ approximation is valid when $\Gamma_{L}+$ $\Gamma_{R} \ll \min \{1 / \tau, \omega, \Delta\}$. In particular, the condition $\Gamma_{L}+\Gamma_{R} \ll 1 / \tau$ implies that the tunneling has a negligible influence on the quasiparticle states; in fact, the only role of the leads is to generate a non-equilibrium quasiparticle population, and the calculation of the $\mathrm{AC}$ response does not depend on the specific way in which a non-equilibrium population is established (as long as it does not substantially alter the quasiparticle states).

Equation (55) can be solved for a generic relation between $1 / \tau$ and $\Delta-$ see Appendix @ Here we consider the dirty limit $\tau \Delta \ll 1$. To calculate the current, we only need the following expression for the Keldysh component:

$$
\begin{aligned}
& \hat{G}_{1}^{K}=i e \mathbf{v} \mathbf{A}_{\omega} \tau \\
& \times\left[\hat{G}_{+}^{R} \hat{\tau}_{z}\left(\hat{G}_{-}^{R}-\hat{G}_{-}^{A}\right) \hat{F}_{-}+\hat{F}_{+}\left(\hat{G}_{+}^{R}-\hat{G}_{+}^{A}\right) \hat{\tau}_{z} \hat{G}_{-}^{A}\right]
\end{aligned}
$$

with $\hat{F}_{ \pm}=\hat{F}(\varepsilon \pm \omega / 2)$. The retarded and advanced Green's functions in this equation are given by Eqs. (13)(14). Substituting Eq. (57) into Eq. (52), we arrive at

$$
\mathbf{j}_{\omega}=\sigma(\omega) \mathbf{E}_{\omega},
$$

where $\sigma(\omega)$ is the complex conductivity. It is convenient to isolate its equilibrium zero-temperature kinetic part

$$
\sigma_{0}(\omega)=-\frac{\pi \sigma_{N} \Delta}{i \omega},
$$

which represents the purely inductive response of the superconducting condensate to the external field in the absence of quasiparticles and where $\sigma_{N}=2 e^{2} \nu_{S} D$ is the normal-state conductivity of the superconductor, with $D=v_{F}^{2} \tau / 3$ the diffusion constant. Using the relationship Eq. (36) between matrix and quasiparticle distribution functions, assuming $0<\omega<2 \Delta$, and performing simple rearrangements, we find

$$
\begin{aligned}
& \sigma(\omega)=\sigma_{0}(\omega)+\frac{2 \sigma_{N}}{\omega} \\
& \times\left[\int_{\Delta}^{\infty} d \varepsilon \frac{\varepsilon(\varepsilon+\omega)+\Delta^{2}}{\sqrt{\left(\varepsilon^{2}-\Delta^{2}\right)\left[(\varepsilon+\omega)^{2}-\Delta^{2}\right]}} f_{\varepsilon}^{E}\right. \\
& \quad-i \int_{\Delta}^{\Delta+\omega} d \varepsilon \frac{\varepsilon(\varepsilon-\omega)+\Delta^{2}}{\sqrt{\left(\varepsilon^{2}-\Delta^{2}\right)\left[\Delta^{2}-(\varepsilon-\omega)^{2}\right]}} f_{\varepsilon}^{E} \\
& \left.-\int_{\Delta+\omega}^{\infty} d \varepsilon \frac{\varepsilon(\varepsilon-\omega)+\Delta^{2}}{\sqrt{\left(\varepsilon^{2}-\Delta^{2}\right)\left[(\varepsilon-\omega)^{2}-\Delta^{2}\right]}} f_{\varepsilon}^{E}\right] .
\end{aligned}
$$

Equation (60) may be viewed as a generalization of the Mattis-Bardeen formula to an arbitrary quasiparticle distribution function $f_{\xi}$.

We now use Eq. (60) to find the AC conductivity for the biased N-I-S-I-N structure considered in Sec. III, concentrating on the limit of low temperatures and voltages, $\max \left\{e V_{L}, e V_{R}, T\right\} \ll \Delta$. In this limit, $f_{\varepsilon}^{E}$ is exponentially small and approximately given by Eq. (38). If in 
addition to the assumptions of low temperature and voltages made above, the frequency is also much smaller than the gap, $\omega \ll \Delta$, we find at leading order in $\Delta$

$$
\begin{array}{r}
\sigma(\omega)=\frac{\sigma_{N} \Delta}{i \omega}\left\{-\pi+2 e^{-\Delta / T} \Phi(e V / T)\right. \\
\times\left[\pi \exp (-\omega / 2 T) I_{0}(\omega / 2 T)\right. \\
\left.\left.+2 i \sinh (\omega / 2 T) K_{0}(\omega / 2 T)\right]\right\},
\end{array}
$$

where $I_{0}$ and $K_{0}$ denote the modified Bessel functions of zeroth order.

The effect of the finite bias on the AC response of the superconductor described by Eq. (61) is contained in full in the function $\Phi$, Eq. (39). In fact, as we now show, this function accounts for the non-equilibrium density of quasiparticles. The quasiparticle density is given by [cf. Eq. [32)]

$$
n_{q p}=2 \nu_{S} \int d \xi f(\xi)=4 \nu_{S} \int_{\Delta}^{\infty} d \varepsilon \frac{\varepsilon}{\sqrt{\varepsilon^{2}-\Delta^{2}}} f_{\varepsilon}^{E}
$$

with $f^{E}$ defined in Eq. (34) and the factor 2 accounting for spin. At low temperature and voltages, we can approximate $f^{E}$ as [see Eq. (38)]

$$
f_{\varepsilon}^{E} \simeq \Phi(e V / T) e^{-\varepsilon / T}
$$

and evaluating the integral at leading order in $\Delta$ we arrive at

$$
n_{q p}=4 \nu_{S} \Phi(e V / T) \sqrt{\frac{\pi \Delta T}{2}} e^{-\Delta / T} .
$$

Using this equation, Eq. (61) can be written as

$$
\begin{aligned}
\sigma(\omega) & =\sigma^{\prime}(\omega)+i \sigma^{\prime \prime}(\omega), \\
\sigma^{\prime}(\omega) & =\sigma_{N} \frac{2 \Delta}{\omega} \sinh \frac{\omega}{2 T} K_{0}\left(\frac{\omega}{2 T}\right) \sqrt{\frac{\Delta}{2 \pi T}} \frac{n_{q p}}{\nu_{S} \Delta} \\
\sigma^{\prime \prime}(\omega) & =\sigma_{N} \frac{\pi \Delta}{\omega}\left[1-e^{-\omega / 2 T} I_{0}\left(\frac{\omega}{2 T}\right) \sqrt{\frac{\Delta}{2 \pi T}} \frac{n_{q p}}{\nu_{S} \Delta}\right] .
\end{aligned}
$$

We note that the above result for the AC conductivity in terms of the quasiparticle density preserves its form even if we include the correction to the distribution function due to the electron-phonon interaction [assuming the condition Eq. (50) is satisfied]. Of course, the quasiparticle density Eq. (64) should be corrected to account for Eq. (49). Equation (65) agrees with the results obtained in Ref. 9 via a phenomenological generalization of the Mattis-Bardeen formula that includes an effective chemical potential for quasiparticles.

\section{SUMMARY AND DISCUSSION}

In this work we have derived an extension of the Mattis-Bardeen formula for the AC conductivity of a dirty superconductor to include a non-equilibrium occupation of the quasiparticle states. Equation (60) shows that the charge imbalance does not affect the linear response to external radiation.

As an explicit example, we considered properties of a superconductor connected via tunnel junctions to two normal leads. Application of a constant bias to the leads creates a steady non-equlibrium state in the superconductor, see Sec. III. We have evaluated bulk AC conductivity $\sigma(\omega)$ of such N-I-S-I-N setup for low frequency $\omega \ll \Delta$ of a weak external field and low temperature $(T \ll \Delta)$ while keeping fixed the DC bias $V$. The role of the finite bias is to create a non-equilibrium quasiparticle density $n_{q p}$. Remarkably, it is possible to express the conductivity $\sigma(\omega)$ as a function of temperature and $n_{q p}$ only, see Eq. (65). The dependence of $\sigma(\omega)$ on bias $V$ enters only through the $V$-dependence of $n_{q p}$. That simplification works even if electron-phonon relaxation is taken into account [if the condition Eq. (50) is satisfied]. At zero bias $(V=0)$ the distribution $n_{q p}$ becomes the equilibrium distribution function, and Eq. 655) reduces to the conventional Mattis-Bardeen formula.

As a further extension one may include the electronelectron interaction. If sufficiently strong, it would lead to the replacement of temperature $T$ in the quasiparticle distribution function with an effective temperature $T_{\text {eff }}$. Interestingly, for a fixed non-equilibrium quasiparticle density, an increase of temperature (or effective temperature) leads to a lower dissipation.

\section{Acknowledgments}

We are grateful to M. Devoret, L. Frunzio, S.M. Girvin, D.S. Prober, and R.J. Schoelkopf for stimulating discussions. KEN thanks Yale University for the hospitality. His visit was made possible by the gift of Victor and Marina Vekselberg to Yale University. This work was supported by IARPA under ARO Contract No. W911NF09-1-0369 (LIG) and Yale University (GC).

\section{Appendix A: Solution to Eq. (55)}

In this Appendix we give some details on how to solve to Eq. (55). We begin with the equation for $\hat{G}_{1}^{R}$

$$
\begin{aligned}
-i \hat{H}_{+} \hat{G}_{1}^{R}+i \hat{G}_{1}^{R} \hat{H}_{-}+ & \frac{1}{2 \tau} \hat{G}_{+}^{R} \hat{G}_{1}^{R}-\frac{1}{2 \tau} \hat{G}_{1}^{R} \hat{G}_{-}^{R}= \\
& -i e \mathbf{v} \mathbf{A}_{\omega}\left(\hat{G}_{+}^{R} \hat{\tau}_{z}-\hat{\tau}_{z} \hat{G}_{-}^{R}\right) .
\end{aligned}
$$

Expressing $\hat{H}_{ \pm}$in Eq. (A1) in terms of $\hat{G}_{ \pm}^{R}$ by means of Eq. (14) and making use of the retarded component of the orthogonality condition (53)

$$
\hat{G}_{+}^{R} \hat{G}_{1}^{R}+\hat{G}_{1}^{R} \hat{G}_{-}^{R}=0
$$


we bring Eq. A1 to the form

$$
\begin{array}{r}
\left(-i \xi_{+}^{R}-i \xi_{-}^{R}+1 / \tau\right) \hat{G}_{+}^{R} \hat{G}_{1}^{R}= \\
-i e \mathbf{v} \mathbf{A}_{\omega}\left(\hat{G}_{+}^{R} \hat{\tau}_{z}-\hat{\tau}_{z} \hat{G}_{-}^{R}\right)
\end{array}
$$

with the obvious notation [cf. Eq. (20)]

$$
\xi_{ \pm}^{R}=\left[\left(\varepsilon \pm \omega / 2+i 0^{+}\right)^{2}-\Delta^{2}\right]^{1 / 2} .
$$

Multiplying both sides of Eq. A3 by $\hat{G}_{+}^{R}$ and making use of the normalization condition $\left(G_{0}^{R}\right)^{2}=1$, we arrive at

$$
\hat{G}_{1}^{R}=\frac{e \mathbf{v} \mathbf{A}_{\omega}}{\xi_{+}^{R}+\xi_{-}^{R}+i / \tau}\left(\hat{\tau}_{z}-\hat{G}_{+}^{R} \hat{\tau}_{z} \hat{G}_{-}^{R}\right) .
$$

Consider now the equation for $\hat{G}_{1}^{K}$ :

$$
\begin{aligned}
& -i \hat{H}_{+} \hat{G}_{1}^{K}+i \hat{G}_{1}^{K} \hat{H}_{-}+\frac{1}{2 \tau}\left(\hat{G}_{+}^{R} \hat{G}_{1}^{K}-\hat{G}_{1}^{K} \hat{G}_{-}^{A}\right) \\
& =-i e \mathbf{v} \mathbf{A}_{\omega}\left(\hat{G}_{+}^{K} \hat{\tau}_{z}-\hat{\tau}_{z} \hat{G}_{-}^{K}\right)-\frac{1}{2 \tau}\left(\hat{G}_{+}^{K} \hat{G}_{1}^{A}-\hat{G}_{1}^{R} \hat{G}_{-}^{K}\right)
\end{aligned}
$$

and the corresponding orthogonality condition

$$
\hat{G}_{+}^{R} \hat{G}_{1}^{K}+\hat{G}_{+}^{K} \hat{G}_{1}^{A}+\hat{G}_{1}^{R} \hat{G}_{-}^{K}+\hat{G}_{1}^{K} \hat{G}_{-}^{A}=0 .
$$

It is convenient to separate $\hat{G}_{1}^{K}$ into a "regular" part, similar in form to Eq. (35), and an "anomalous" one as follows:

$$
\hat{G}_{1}^{K}=\hat{G}_{1}^{r}+\hat{G}_{1}^{a}, \quad \hat{G}_{1}^{r}=\hat{G}_{1}^{R} \hat{F}_{-}-\hat{F}_{+} \hat{G}_{1}^{A},
$$

where $\hat{F}_{ \pm}=\hat{F}(\varepsilon \pm \omega / 2)$. A substitution of this definition into the orthogonality condition (A7) yields the much simpler equation

$$
\hat{G}_{+}^{R} \hat{G}_{1}^{a}+\hat{G}_{1}^{a} \hat{G}_{-}^{A}=0 .
$$

Substituting Eq. (A8) into Eq. (A6) and using Eq. (A1) we find the equation for $\hat{G}_{1}^{a}$

$$
\begin{aligned}
& -i \hat{H}_{+} \hat{G}_{1}^{a}+i \hat{G}_{1}^{a} \hat{H}_{-}+\frac{1}{2 \tau}\left(\hat{G}_{+}^{R} \hat{G}_{1}^{a}-\hat{G}_{1}^{a} \hat{G}_{-}^{A}\right) \\
& =-i e \mathbf{v} \mathbf{A}_{\omega}\left[\hat{G}_{+}^{R}\left(\hat{F}_{+} \hat{\tau}_{z}-\hat{\tau}_{z} \hat{F}_{-}\right)-\left(\hat{F}_{+} \hat{\tau}_{z}-\hat{\tau}_{z} \hat{F}_{-}\right) \hat{G}_{-}^{A}\right] .
\end{aligned}
$$

The solution to this equation can be found by following the procedure similar to that used above to solve for $\hat{G}_{1}^{R}$. First, we express $\hat{H}_{+}$and $\hat{H}_{-}$in terms of $\hat{G}_{+}^{R}$ and $\hat{G}_{-}^{A}$, respectively:

$$
\begin{aligned}
& \left(-i \xi_{+}^{R}+\frac{1}{2 \tau}\right) \hat{G}_{+}^{R} \hat{G}_{1}^{a}-\left(-i \xi_{-}^{A}+\frac{1}{2 \tau}\right) \hat{G}_{1}^{a} \hat{G}_{-}^{A} \\
& =-i e \mathbf{v} \mathbf{A}_{\omega}\left[\hat{G}_{+}^{R}\left(\hat{F}_{+} \hat{\tau}_{z}-\hat{\tau}_{z} \hat{F}_{-}\right)-\left(\hat{F}_{+} \hat{\tau}_{z}-\hat{\tau}_{z} \hat{F}_{-}\right) \hat{G}_{-}^{A}\right] .
\end{aligned}
$$

Then, using the orthogonality condition Eq. (A9), we eliminate $\hat{G}_{-}^{A}$ in favor of $\hat{G}_{+}^{R}$ :

$$
\begin{aligned}
& \left(-i \xi_{+}^{R}-i \xi_{-}^{A}+1 / \tau\right) \hat{G}_{+}^{R} \hat{G}_{1}^{a}=-i e \mathbf{v} \mathbf{A}_{\omega} \\
& \quad \times\left[\hat{G}_{+}^{R}\left(\hat{F}_{+} \hat{\tau}_{z}-\hat{\tau}_{z} \hat{F}_{-}\right)-\left(\hat{F}_{+} \hat{\tau}_{z}-\hat{\tau}_{z} \hat{F}_{-}\right) \hat{G}_{-}^{A}\right]
\end{aligned}
$$

The solution of this equation is obtained by multiplying both sides by $\hat{G}_{+}^{R}$ and employing the normalization condition $\left(\hat{G}_{0}^{R}\right)^{2}=1$ :

$$
\begin{aligned}
\hat{G}_{1}^{a} & =\frac{e \mathbf{v} \mathbf{A}_{\omega}}{\xi_{+}^{R}+\xi_{-}^{A}+i / \tau} \\
& \times\left[\hat{F}_{+} \hat{\tau}_{z}-\hat{\tau}_{z} \hat{F}_{-}-\hat{G}_{+}^{R}\left(\hat{F}_{+} \hat{\tau}_{z}-\hat{\tau}_{z} \hat{F}_{-}\right) \hat{G}_{-}^{A}\right] .
\end{aligned}
$$

Equations A5 and A13 are valid for $\Gamma_{i} \ll \Delta, 1 / \tau$ and arbitrary relation between $\Delta$ and $1 / \tau$. In the dirty limit $\Delta \tau \ll 1$, Eqs. (A5) and (A13) can be respectively approximated as

$$
\begin{aligned}
\hat{G}_{1}^{R} & =-i e \mathbf{v} \mathbf{A}_{\omega} \tau\left(\hat{\tau}_{z}-\hat{G}_{+}^{R} \hat{\tau}_{z} \hat{G}_{-}^{R}\right), \\
\hat{G}_{1}^{a} & =-i e \mathbf{v} \mathbf{A}_{\omega} \tau \\
& \times\left[\hat{F}_{+} \hat{\tau}_{z}-\hat{\tau}_{z} \hat{F}_{-}-\hat{G}_{+}^{R}\left(\hat{F}_{+} \hat{\tau}_{z}-\hat{\tau}_{z} \hat{F}_{-}\right) \hat{G}_{-}^{A}\right] .
\end{aligned}
$$

Substituting these equations into Eq. (A8) we find Eq. (57).
1 J. M. Ziman, Principles of the Theory of Solids (CUP, Cambridge, 1979).

2 M. Tinkham, Introduction to superconductivity (McGrawHill, New York, 1996).

3 D. N. Basov and T. Timusk, Rev. Mod. Phys. 77, 721 (2005).

4 O. V. Dolgov, A. A. Golubov, and D. Parker, New J. Phys. 11, 075012 (2009).

5 A. Blais, R.-S. Huang, A. Wallraff, S. M. Girvin, and R. J. Schoelkopf, Phys. Rev. A 69, 062320 (2004).

6 A. Wallraff, D. I. Schuster, A. Blais, L. Frunzio, R.-S.
Huang, J. Majer, S. Kumar, S. M. Girvin and R. J. Schoelkopf, Nature 431, 162 (2004).

7 S. Gleyzes, S. Kuhr, C. Guerlin, J. Bernu, S. Deleglise, U. B. Hoff, M. Brune, J.-M. Raimond, and S. Haroche, Nature 446, 297 (2007).

8 S. Kuhr, S. Gleyzes, C. Guerlin, J. Bernu, U. B. Hoff, S. Deleglise, S. Osnaghi, M. Brune, J.-M. Raimond, S. Haroche, E. Jacques, P. Bosland, and B. Visentin, Appl. Phys. Lett. 90, 164101 (2007).

9 J. Gao, J. Zmuidzinas, A. Vayonakis, P. Day, B. Mazin, and H. Leduc, J. Low Temp. Phys. 151, 557 (2008). 
10 D. C. Mattis and J. Bardeen, Phys. Rev. 111, 412 (1958).

11 A. A. Abrikosov, L. P. Gorkov, and I. M. Khalatnikov, Zh. Eksperim. i Teor. Fiz. 35, 265 (1958) [Sov. Phys. JETP 8, $182(1958)]$

12 S. B. Nam, Phys. Rev. 156, 470 (1967); Phys. Rev. 156, 487 (1967).

13 See, e.g., J. P. Turneaure and I. Weissman, J. Appl. Phys. 39, 4417 (1968), and references therein.

14 R. Barends, J. J. A. Baselmans, S. J. C. Yates, J. R. Gao, J. N. Hovenier, and T. M. Klapwijk, Phys. Rev. Lett. 100, 257002 (2008).

15 For a review of the Keldysh approach see, e.g., Ref. 16.

16 J. Rammer and H. Smith, Rev. Mod. Phys. 58, 323 (1986).

17 I. Snyman and Yu. V. Nazarov, Phys. Rev. B 79, 014510
(2009).

18 More precisely, in Eqs. (43)-(44) we use for $1 / \tau_{p h}$ the definition of Ref. 20, which differs from the standard one ${ }^{19}$ by the numerical factor $\left(T_{c} / \Delta_{0}\right)^{3}$. Since we are interested in the low-temperature, low-bias regime, we neglect the difference between the gap $\Delta$ and its zero-temperature, equilibrium value $\Delta_{0}$.

19 S. B. Kaplan, C. C. Chi, D. N. Langenberg, J. J. Chang, S. Jafarey, and D. J. Scalapino, Phys. Rev. B 14, 4854 (1976).

20 V. M. Galitskii, V. F. Elesin, and Yu. V. Kopaev, in Nonequilibrium superconductivity, D. N. Langenberg and A. I. Larkin eds. (North Holland, Amsterdam, 1986). 